\title{
Ações preventivas em enfermagem ao HIV/AIDS na atenção primária de saúde
}

\author{
Preventive actions in HIV/AIDS nursing in primary health care \\ Acciones preventivas en enfermería VIH/SIDA en atención primaria de salud
}

Elaine Freitas de Araújo ${ }^{1 *}$ Jaqueline Moreira de Albuquerque ${ }^{1}$, Nelciene da Silva Guimarães Farias ${ }^{1}$, Jabneela Vieira Pereira Vetorazo ${ }^{1}$.

\section{RESUMO}

Objetivo: Esclarecer como vem ocorrendo as ações preventivas em enfermagem ao HIV/AIDS na Atenção Primária de Saúde (APS). Métodos: Revisão integrativa da literatura, de abordagem qualitativa/qualitativa, que tomou por bases de dados a Biblioteca Virtual em Saúde (BVS), bem como a Biblioteca Eletrônica Científica Online (Scielo) e a Acervo+, no espaço temporal entre 2016 a 2021, que discutissem os aspectos relacionados às ações de prevenção ao HIV na APS. Resultados: Encontraram-se 124 artigos nas bases de dados, e após a leitura dos títulos foram separados 92 artigos que passaram pela análise dos critérios de inclusão, sendo escolhidos 32 para leitura na íntegra e ao final foram selecionados 14 artigos para subsidiar a presente revisão de literatura. Considerações Finais: Os(as) enfermeiro(as) na APS atuam de maneira basilar no processo de prevenção de doenças, pois, podem criar estratégias para sensibilizar, informar e empoderar os pacientes e assim diminuir a exposição ao HIV/AIDS, por meio de atividades como rodas de conversa, palestras, execução de testes rápidos, educação em saúde, campanhas, entre outros.

Palavras-chave: Enfermagem, HIV/AIDS, Adolescentes, Atenção primária a saúde.

\begin{abstract}
Objective: To clarify how preventive actions in nursing against HIV/AIDS have been taking place in Primary Health Care (PHC). Methods: Integrative literature review, qualitative / qualitative approach, based on databases in the Virtual Health Library (VHL), as well as in the Online Scientific Electronic Library (Scielo) and Acervo +, in the period between 2016 to 2021, to discuss aspects related to HIV prevention actions in PHC. Results: 124 articles were found in the databases, and after reading the separate titles, 92 articles that went through the analysis of the inclusion criteria, 32 were chosen for full reading and, in the end, 14 articles were selected to support this review of literature. Final Considerations: The nurses in the PHC act in a fundamental way in the disease prevention process, as they can create strategies to sensitize, inform and empower patients and thus reduce exposure to HIV / AIDS, through activities such as conversation circles, lectures, running quick tests, health education, campaigns, among others.
\end{abstract}

Key words: Nursing, HIV/AIDS, Adolescents, Primary health care.

\section{RESUMEN}

Objetivo: Aclarar cómo se vienen desarrollando las acciones preventivas en enfermería frente al VIH / SIDA en la Atención Primaria de Salud (APS). Métodos: Revisión bibliográfica integradora, abordaje cualitativo / cualitativo, con base en bases de datos en la Biblioteca Virtual en Salud (BVS), así como en la Biblioteca Electrónica Científica en Línea (Scielo) y Acervo +, en el período comprendido entre 2016 a 2021, para discutir aspectos relacionados con las acciones de prevención del VIH en la APS. Resultados: Se encontraron 124 artículos en las bases de datos, y luego de la lectura de los títulos separados, 92 artículos que pasaron por el análisis de los criterios de inclusión, 32 fueron seleccionados para lectura completa y, al final, 14 artículos fueron seleccionados para apoyar esta revisión de literatura. Consideraciones finales: Las enfermeras de la APS actúan de manera fundamental en el proceso de prevención de la enfermedad, ya que pueden crear estrategias para sensibilizar, informar y empoderar a los pacientes y así reducir la exposición al VIH / SIDA, a través de actividades como círculos de conversación, charlas, carreras. pruebas rápidas, educación en salud, campañas, entre otros.

Palabras clave: Enfermería, VIH/SIDA, Adolescentes, Atención primaria de salud.

${ }^{1}$ Centro Universitário Aparício Carvalho (FIMCA), Porto Velho - RO. *E-mail: laineaa23@gmail.com

SUBMETIDO EM: 10/2021

ACEITO EM: 10/2021

PUBLICADO EM: 10/2021 


\section{INTRODUÇÃO}

O HIV é uma sigla que define o vírus da imunodeficiência humana em sua tradução em inglês significa "Human Immunodeficiency Virus". O mesmo é um problema de saúde pública devido sua gravidade e magnitude. Entretanto não existe cura para o mesmo, além de que o vírus não faz distinção de cor, raça, sexo ou idade, todos são propícios a adquirir a doença, a cada dia os casos vem se alarmando ainda mais (SANTOS CP, et al., 2010; MINISTÉRIO DA SAÚDE, 2017).

Em 1982 foi o relatado o primeiro caso de Síndrome da Imunodeficiência Adquirida (AIDS) no Brasil (CASTELLANI MMX e MORETTO MLT, 2016). A partir da década de 80, houve uma disseminação da AIDS no mundo, representando a pandemia da atualidade, haja vista que, em 2017, cerca de 36,9 milhões de pessoas em todo o mundo viviam com HIV, conforme o Programa Conjunto das Nações Unidas sobre HIV/AIDS (UNIAIDS), no ano de 2019.

Acredita-se que cerca de 886 mil pessoas vivem com HIV no país, sendo a AIDS uma doença de relevância para a saúde pública, em vista disso o Ministério da Saúde (MS) preconizou que o HIV e a AIDS, quando diagnosticado deve ser notificado imediatamente, sendo de caráter compulsório desde 2014 (GALANO E, et al., 2016). De acordo com o Ministério da Saúde (2020), o Brasil vem registrando uma queda no número de casos de infecção por Aids de 2012 a 2019, como um índice de decréscimo de 18,7\%. Em relação ao índice de mortalidade por Aids caiu 17,1\%. As quedas são justificadas pelos movimentos de testagem rápida realizado pelos(as) enfermeiros(as) na APS, o que possibilita o início de terapia imediata, quando paciente é diagnosticado como soro positivo.

O Manual técnico disponibilizado pelo MS para diagnosticar a infecção pelo HIV, indica dentre as estratégias, a testagem rápida. A mesma tem a finalidade de aprimorar a qualidade do diagnóstico da infecção pelo HIV apontando se é recente ou não, e além de alimentar de dados o sistema nacional. Salienta-se que trata-se de um exames seguros e que possuem a vantagem de ser concluído de forma célere (MINISTÉRIO DA SAÚDE, 2017). Ainda, de acordo com o MS, devem ser planejadas e organizadas capacitações pelos gestores estaduais para os profissionais de Atenção Básica direcionadas a execução dos testes rápidos de HIV e sífilis, para que os mesmos alimentem o sistema de notificações, com fins de monitorar e criar estratégias de reduzir as taxas de transmissão vertical do HIV e a eliminação da sífilis congênita, bem como a reduzir a mortalidade materna e infantil (MINISTÉRIO DA SAÚDE, 2021a).

Portanto, diagnosticar e acompanhar as pessoas que vivem com o vírus, ainda é desafiador para a reorganização da Rede de Atenção em Saúde (RAS), especialmente em relação as realidades dos profissionais da APS, na qual, geralmente, há déficits no que tange a assistência quando há necessidade de exames, por falta muitas vezes de insumos e materiais para coleta e análise. Além disso, os profissionais precisam lidar com a revelação de resultados positivos, dando um suporte aos pacientes e seus familiares na desestigmatização de preconceitos sofridos pelos usuários, sendo necessário maior capacitação para esses profissionais da enfermagem, os quais são também responsáveis por facilitar o processo de aceitação (LIMA MCL, et al., 2020).

Além de todos esses esforços para reduzir índice de contaminação e óbitos por AIDS, o Ministério da Saúde fornece medicação gratuita aos pacientes soro positivos e vem promovendo Campanhas de prevenção ao HIV/Aids, geralmente fomentadas no "Dia Mundial de Luta Contra a Aids" que se celebra no dia 1 de Dezembro. A campanha faz uso das mídias sociais e veículos de comunicação para incentivar uso de preservativo, a testagem rápida, bem como, o fim do preconceito contra pessoas soropositivas (MINISTÉRIO DA SAÚDE, 2020).

Nesse contexto, os pacientes procuram o serviço de saúde principalmente pela APS. As portas do Sistema de Saúde atuam com a grande responsabilidade de acolher e ter uma escuta qualificada sobre as necessidades da população e direcionar os usuários de maneira humanizada, organizando o fluxo do atendimento (MELO EA, et al., 2018).

De acordo com a Organização Mundial da Saúde (OMS) (2020), o conceito de atenção primária à saúde está em constante redefinição, pois, precisa se adequar para estar de acordo com três objetivos: a garantia 
de acesso a serviços voltados a prevenir, promover, educar, proteção, curar, reabilitar e cuidar com foco no indivíduo e a família; Buscar meios de atuar conforme as necessidade da população, considerando os aspectos sociais, econômicos e ambientais; capacitar e sensibilizar a comunidade atendida de forma a Ihes passar as orientações necessárias, no âmbito da saúde, que thes empoderem de conhecimento sobre as enfermidades e como se prevenir e otimizar sua saúde.

De acordo com o MS (2020b), no Brasil, o processo de enfretamento da epidemia de HIV/aids se iniciou com a oferta gratuita da Terapia Antirretroviral (TARV) a todos os usuários do Sistema único de Saúde (SUS), de acordo com a Lei no 9.313, de 13 de novembro de 1996. Já em 2007, o país liderou o mundo com licenciamento compulsório das patentes de medicamentos antirretrovirais. O Brasil ainda se destaca a nível mundial por promover ações de prevenção, além da testagem em massa que acontece na rede de saúde de forma estratégica busca fortalecer a Atenção Primária de Saúde (APS) para atuar nesse sentido, possibilitando maior cobertura e impedindo que a epidemia alcance maior índice de pessoas.

Nesse contexto, (a) enfermeiro (a) assume um papel de destaque ao liderar equipes de saúde da família, que atuam juntos aos usuários da APS, visando prevenir, diagnosticar e acompanhar os mesmos, realizando a vigilância epidemiológica, tanto coletiva como individual, e quando houver necessidade realizando Projetos Terapêuticos Singulares, com o escopo de proporcionar através de sua capacitação, o planejamento e organizando ações preventivas adequadas ao público adstrito, sendo o principal articulador, além de ser responsável por fomentar e realizar testes rápidos e notificação dos casos (MINISTÉRIO DA SAÚDE, 2017).

O trabalho do (a) enfermeiro (a) no âmbito da APS é elementar, haja vista suas funções preventivas, como coletor de exames (preventivo do colo uterino, teste rápido, entre outros), e a versatilidade necessária para atuar em educação em saúde de forma dinâmica, inovadora, criativa, e assim prevenir doenças, ofertar cuidado, acolher, ouvir, direcionar, orientar, encaminhar, acompanhar, monitorar (TUMÉ E, et al., 2018). Todas essas funções muitas vezes desempenhadas em áreas de difícil acesso, para populações vulneráveis, e em grande parte sem os recursos necessários para melhor atender, sendo assim reconhecida sua importância profissional pela Organização Mundial da Saúde (OMS, 2016).

Além de todas as importantes funções do enfermeiro na APS, destaca-se o diagnóstico realizado no prénatal, e no que tange a AIDS, ele atua em acordo com as políticas de enfrentamento, realizando os exames para reconhecer de forma precoce possível infecção pelo HIV durante a gestação, e assim, o profissional adota importantes ações profiláticas que minimizam a transmissão da mãe para o bebê, que pode ocorrer na hora do parto ou durante a amamentação. Nesse momento o(a) enfermeiro(a) precisa manter um relacionamento de confiança com a mãe soro positiva para que ela continue o pré-natal, e contribua para evitar a transmissão para a criança (PORTELA LMSR, et al., 2021).

Diante do enunciado, o presente estudo teve como objetivo esclarecer como vem ocorrendo as ações preventivas em enfermagem ao HIV/AIDS na APS. Os resultados podem oferecer informações importantes para que se intervenha nesta situação, a fim de prestar assistência de excelência.

\section{MÉTODOS}

Este estudo é uma revisão de literatura baseada em artigos publicados na literatura até Agosto de 2021. O qual segue 6 passos: se formulou primeiramente a questão norteadora da pesquisa, depois, foi coletada a literatura pertinente a temática, foram extraídos os dados, avaliadas as publicações, interpretados os resultados e, finalmente apresentada a revisão integrativa. Este método reúne os resultados de pesquisas sobre um delimitado tema, visando aprofundar o conhecimento do tema averiguado (MENDES KDS, et al., 2008).

A questão central que norteou esse estudo foi: "Como vem ocorrendo vem ocorrendo as ações preventivas em enfermagem ao HIVIAIDS, na APS?" A Coleta de dados e tabulação dos dados foi feita por meio de um quadro de informações elaborado através do programa Microsoft Excel contendo os elementos. Ao coletar todo o material, foram avaliados os estudos incluídos na revisão integrativa através de uma leitura crítica. Os 
bancos de dados utilizados para busca foram: Biblioteca Virtual em Saúde (BVS), em Acervo+, e na Biblioteca Eletrônica Científica Online (SCIELO).

Tomou-se por critério de seleção a inclusão de artigos científicos disponíveis na íntegra, publicados no espaço temporal entre 2016 a 2021, em língua portuguesa e que após lidos integralmente, estariam em conformidade com os objetivos propostos. Excluíram-se os artigos duplicados, incompletos, artigos de revisão, teses etc. Ao interpretar e sintetizar os resultados, ocorreu a interpretação dos mesmos e a discussão, e organizaram-se os resultados em uma tabela, contendo autores e ano, periódico, e a abordagem relacionada a assistência preventiva de enfermagem direcionada ao HIV, na APS

\section{RESULTADOS}

Após a busca nas bases de dados e excluídos artigos que não compreendia o período de 2016 a 2021 , foram selecionados 124 artigos, sendo 66 artigos na base de dados da BVS, 54 artigos na base de dados da Scielo e 4 artigos na base de dados da Acervo +. Após a leitura dos títulos foram separados 92 artigos que passaram pela análise dos critérios de inclusão, sendo excluídos 15 artigos por estarem indisponíveis; 16 por serem datados o período inferior ao selecionado; 15 por estarem em outro idioma; 15 publicações por serem teses; 13 publicações por apresentarem somente título e resumo e 18 por estarem duplicados na base de dados.

Após a leitura do resumo, foram selecionados 32 estudos para serem analisados. Esses 32 foram lidos na íntegra e, 14 artigos foram incluídos para a discussão acerca do tema, como mostrado fluxograma a seguir (Figura 1).

Figura 1 - Fluxograma demonstrativo das estratégias de busca nas bases de dados, 2021.

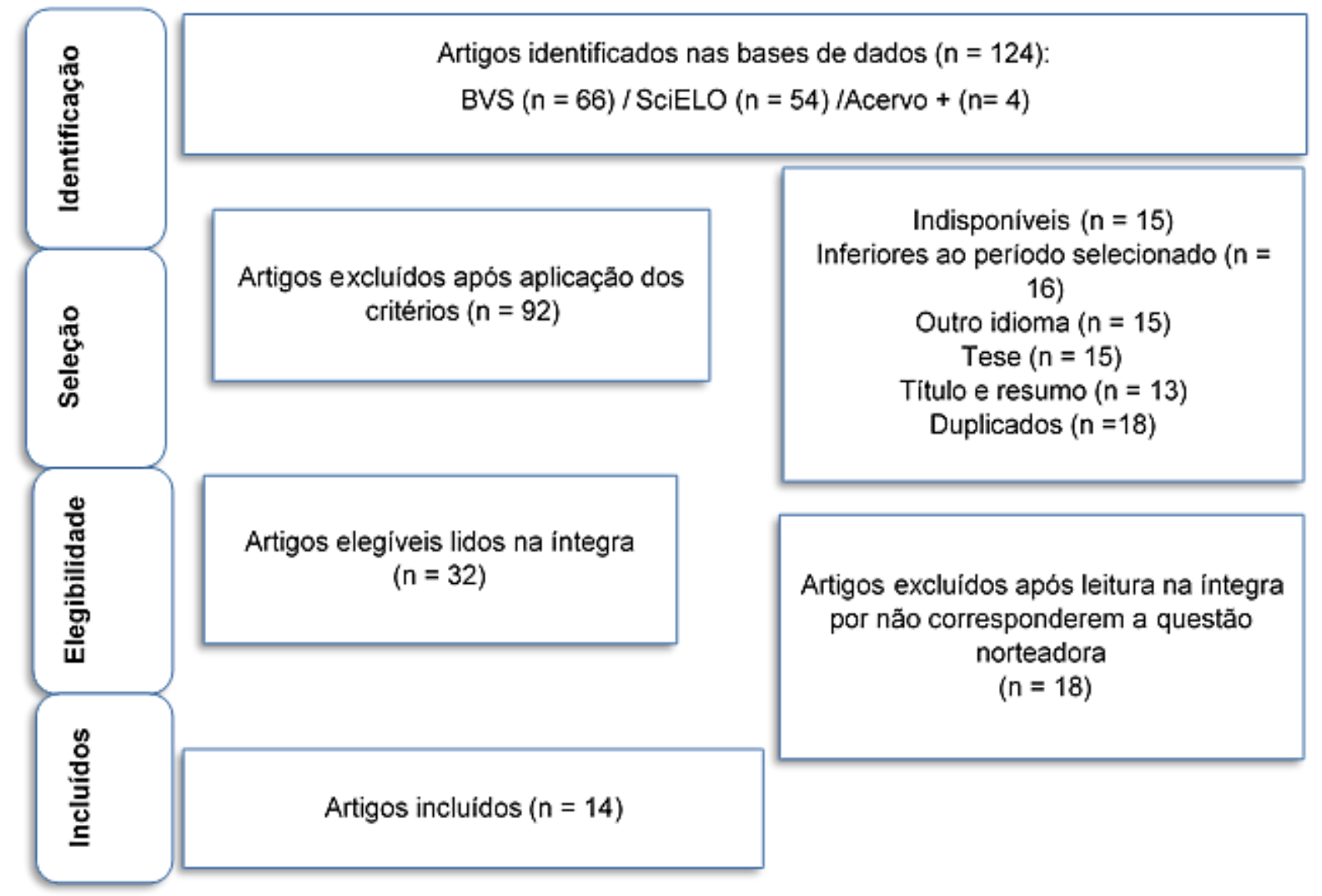

Fonte: Araújo EF, et al., 2021. 
Dentre os oito selecionados e incluídos na Revisão Integrativa a partir da leitura na íntegra, 1 foi publicado em 2017, 5 foram publicados em 2018, 3 foram publicados em 2019, 3 foram publicados em 2020 e 2 foram publicados em 2021. Três estudos foram publicados na revista Esc. Anna Nery e três na Revista Eletrônica Acervo Enfermagem (Quadro 1).

Quadro 1 - Dados dos artigos selecionados para esse estudo: os autores, ano, país e periódico de publicação, tipo de estudo compreendendo as ações preventivas em enfermagem ao HIV/AIDS no âmbito da Atenção Primária de Saúde (APS).

\begin{tabular}{|c|c|c|}
\hline Autores e ano & Periódico & Abordagem \\
\hline $\begin{array}{l}\text { Oliveira JAS e Afonso SM } \\
\text { (2017) }\end{array}$ & Rev. CIE & $\begin{array}{l}\text { O Teste Rápido para o Diagnóstico de HIV na } \\
\text { Atenção Primária à Saúde e a importância da } \\
\text { atuação do Enfermeiro }\end{array}$ \\
\hline Melo EA, et al. (2018) & $\begin{array}{l}\text { Rev. Panar } \\
\text { de Salud Púl }\end{array}$ & $\begin{array}{l}\text { Cuidado, HIV/Aids e atenção primária no Brasil: } \\
\text { desafio para a atenção no Sistema Único de Saúde? }\end{array}$ \\
\hline Almeida Jr JA, et al. (2018) & Rev Baiana Enferm. & $\begin{array}{l}\text { Teste rápido para HIV: representações sociais de } \\
\text { profissionais da atenção básica. }\end{array}$ \\
\hline $\begin{array}{l}\text { Fontinele DCSS, et al. } \\
(2018)\end{array}$ & $\begin{array}{l}\text { Revista Humano Ser } \\
\text { - UNIFACEX }\end{array}$ & $\begin{array}{l}\text { Ações de Enfermagem para Inserção dos } \\
\text { Homossexuais Soropositivos nos Serviços de } \\
\text { Saúde. }\end{array}$ \\
\hline Araújo WJ, et al. (2018) & Rev Bras Enferm. & $\begin{array}{l}\text { Percepção de enfermeiros executores de teste } \\
\text { rápido em Unidades Básicas de Saúde. }\end{array}$ \\
\hline Sant & Anai & $\begin{array}{l}\text { infecções } \\
\text { Básica, }\end{array}$ \\
\hline Sant & Esc. Anna & $\begin{array}{l}\text { Roteiros de sexualidade construídos por enfermeiros } \\
\text { e a interface com a atenção em infecções } \\
\text { sexualmente transmissíveis/HIV }\end{array}$ \\
\hline Ran & $\begin{array}{l}\text { Revista Elet } \\
\text { Acervo Enferm }\end{array}$ & $\begin{array}{l}\text { A educação em saúde como ferramenta estratégica } \\
\text { no desenvolvimento de ações de prevenção da } \\
\text { transmissão do HIV: Um Relato de Experiência. }\end{array}$ \\
\hline Previati SM, & J. Health B & $\begin{array}{l}\text { A importância do aconselhamento no exame rápido } \\
\text { de HIV em gestantes durante o pré-natal }\end{array}$ \\
\hline Pinho CM, e & $\begin{array}{l}\text { Revista Eletr } \\
\text { Acervo Enferma }\end{array}$ & $\begin{array}{l}\text { Avaliação das medidas de controle do HIV na } \\
\text { atenção básica. }\end{array}$ \\
\hline Frei & $\begin{array}{l}\text { Research, Society } \\
\text { and Development }\end{array}$ & $\begin{array}{l}\text { Avaliação do Controle do HIV/Aids na Atenção } \\
\text { Primária em Palmas/TO }\end{array}$ \\
\hline Souz & $\begin{array}{l}\text { Revista Bras } \\
\text { Multidisciplinar }\end{array}$ & $\begin{array}{l}\text { Percepções dos enfermeiros mediante a realização } \\
\text { do teste rápido de HIV/AIDS na atenção primária à } \\
\text { saúde }\end{array}$ \\
\hline So & $\begin{array}{l}\text { Revista Eletrônica } \\
\text { Acervo Enfermagem }\end{array}$ & $\begin{array}{l}\text { Fatores atribuídos a assistência de enfermagem aos } \\
\text { portadores da infecção pelo vírus do HIV/AIDS }\end{array}$ \\
\hline Lim & $E$ & $\begin{array}{l}\text { Percepção dos enfermeiros acerca do processo de } \\
\text { descentralização do atendimento ao HIV/Aids: } \\
\text { testagem rápida }\end{array}$ \\
\hline
\end{tabular}

Fonte: Araújo EF, et al., 2021.

\section{DISCUSSÃO}

Frente a literatura encontrada, pode-se compreender como vem ocorrendo a assistência de enfermagem na prevenção do HIV, na APS. Evidenciou-se que, a prevenção do HIV é um dos pontos abordados pelos enfermeiros dentro da APS, por meio do acolhimento humanizado, consultas de enfermagem, testes rápidos, aconselhamento individual, realização de grupos de adesão conforme a demanda, além dos enfermeiros notificarem os casos realizarem campanhas nacionais anuais, nos períodos de Carnaval e no dia Mundial de Combate à Aids (SANTOS AHF, et al., 2018). Acredita-se que a atenção básica é fundamental para a 
ocorrência da prevenção do HIV, haja vista que, garante maior acesso e vínculo ao sistema de saúde (PINHO CM, et al. 2020).

$\mathrm{O}$ (a) enfermeiro(a) é o profissional capacitado para além de informar os pacientes, aconselhar e para esclarecer sobre o vírus e a doença, de forma que sejam quebrados preconceitos (RAMOS FBP, et al., 2019). Santos AHF, et al. (2018) e Oliveira JAS e Afonso TM (2017), expõem a necessidade do(a) enfermeiro(a) trabalhar as questões espirituais e culturais, pois os preconceitos baseiam-se em crenças de que a AIDS está ligada ao homossexualismo, sexo fora do casamento, medo de se contaminar por meio de abraço e outros estigmas, sendo necessário discutir essas questões de preconceito social, fazendo com que mais pessoas tenham a compressão da infecção, da ação do vírus no organismo.

Desta forma, na APS os enfermeiros trabalham promovendo ações informativas, de conscientização a respeito da forma de transmissão, contágio, sintomas, explicando como funcionam os testes rápidos, e como é o tratamento, trabalhando o aconselhamento de forma humanizada e acolhedora, para criar um elo de confiança com os pacientes, minimizando dúvidas e medos dos usuários (SANTOS AHF, et al., 2018).

Porém, a literatura aponta que, a atuação dos enfermeiros ao tratar a questão da prevenção do HIV/AIDS não se apresenta como deve ser, conforme o preconizado pelo Conselho Federal de Enfermagem e pelo MS, muitas vezes justificam-se pelas limitadas condições de trabalho, visto que precisa atender alta demanda, deixando em segundo plano esse aconselhamento preventivo (SOUZA J, et al. 2021; RAMOS FBP, et al., 2019). Sendo assim, Melo EA, et al. (2018), afirma que cada vez mais se busca por políticas públicas que visem a capacitação dos profissionais da atenção primária, sobretudo o(a) enfermeiro(a), para atuar com maior efetividade na prevenção do HIV/AIDS, a fim de fortalecer a ESF e APS e integrá-las nas Redes de Atenção à Saúde (RAS).

Faz-se necessário políticas de apoio matricial que articule constantemente com as práticas clínicas generalistas oferecidas na APS, de forma a assegurar a continuidade e eficácia da assistência prestada (SOUZA J, et al. 2021; RAMOS FBP, et al., 2019). Lima MCL, et al. (2021) e Júnior JAA, et al. (2018), afirmam que há falhas na responsabilização e comprometimento do enfermeiro com a prevenção do HIV na APS, e esse empecilho limita as estratégias preventivas que poderiam ser implementadas para auxiliar os usuários e assim diminuir ainda mais os índices de infectados e mortes por HIV/AIDS. Neste sentido, Souza J, et al. (2021), confirma, relatando que a prática da prevenção ainda é fraca indicando a necessidade de apoio matricial e especializado.

Frente ao exposto, salienta-se a importância para a prevenção do HIV/AIDS da realização de rodas de conversas entre os "profissionais X profissionais", "profissionais X usuários", para que sejam esclarecidas dúvidas relacionadas ao processo evolutivo da AIDS, isso desde a adolescência quando muitos iniciam as atividades sexuais, logo os profissionais da ESF na APS podem criar estratégias de planejar e organizar palestras nas escolas e levar as informações importantes sobre uso de preservativo, por exemplo, são simples ações que podem contribuir com redução de índice de pessoas HIV/AIDS (SANTOS SMP, et al., 2019)

Pois, entende-se que há necessidade de apoio matricial, buscar parcerias com profissionais da saúde mental para ministrar palestras e esclarecer sobre o tema, bem como profissionais que atuam junto aos pacientes com HIV para esclarecer mais sobre a terapia medicamentosa utilizada, sobre os exames e sobre o serviço de rede oferecido para a população. Há que se fazer também mais investimento na capacitação dos profissionais, a fim de melhorar a assistência preventiva da enfermagem na APS (FREITAS CC, et al., 2020).

Estudos defendem a necessidade da criação de rede de proteção e suporte aos pacientes e familiares com HIV, pois, os não portadores do vírus poderiam reforçar o entendimento sobre a doença e a contaminação pelo vírus, diminuindo medos, tensões e discriminação, além de alcançarem uma visão mais esclarecedora sobre como se prevenir e também disponibilizarem de testes rápidos (PREVIATI SM, et al., 2019; FONTINELE DCSS, et al., 2018; SOUZA LRB, et al., 2020). Araújo WJ, et al. (2018) explica que o estímulo à realização da testagem anti-hiv pode ser avaliada como uma das estratégias para prevenção da transmissão do HIV mais eficazes, pois, minimiza a morbidade e mortalidade. 


\section{CONSIDERAÇÕES FINAIS}

Pode-se considerar que, o âmbito da APS é espaço fundamental para se trabalhar a prevenção do HIV, sendo o enfermeiro peça chave desse processo, e, atualmente, atua na prevenção por meio de acolhimento humanizado, consultas de enfermagem, realização de testes rápidos, aconselhamento individual, realizando notificações, campanhas nacionais anuais, notificações e testes rápidos. Nesse processo, a educação em saúde é o principal instrumento para atuar estrategicamente no desenvolvimento de ações de prevenção da transmissão do HIV, bem como para combater o estigma e preconceito sofrido pelas pessoas que possuem o vírus. O presente estudo contribui como subsídio para outros estudos a respeito da temática, a fim de que, busque-se aprimorar ainda mais a prevenção da infecção por HIV, na APS.

\section{REFERÊNCIAS}

1. ARAÚJO WJ, et al. Percepção de enfermeiros executores de teste rápido em Unidades Básicas de Saúde. Rev Bras Enferm, 2018; 71:631-6.

2. FREITAS CC, et al. Avaliação do Controle do HIV/Aids na Atenção Primária em Palmas/TO. Research, Society and Development, 2020; 9(9): e372997126.

3. FONTINELE DCSS, et al. Ações de Enfermagem para Inserção dos Homossexuais Soropositivos nos Serviços de Saúde. Revista Humano Ser - UNIFACEX, 2018; 3 (1):84-100.

4. GALANO E, et al. Vivências dos adolescentes soropositivos para HIV/Aids: estudo qualitativo. Revista Paulista de Pediatria. 2016; 34 (2):171-177.

5. JÚNIOR JAA, et al. Teste rápido para HIV: representações sociais de profissionais da atenção básica. Rev Baiana Enferm, 2018; 32:e25885.

6. LIMA MCL. Percepção dos enfermeiros acerca do processo de descentralização do atendimento ao HIV/Aids: testagem rápida. Esc. Anna Nery, 2021; 25(4):e20200428.

7. MELO EA, et al. Cuidado, HIV/Aids e atenção primária no Brasil: desafio para a atenção no Sistema Único de Saúde? Revista Panamericana de Salud Pública, 2018; 42: e151.

8. MENDES KDS, et al. Revisão integrativa: método de pesquisa para a incorporação de evidências na saúde e na enfermagem. Texto Contexto Enferm, 2008; 17(4): 758-64.

9. MINISTERIO DA SAÚDE. Casos de Aids diminuem no Brasil: Boletim Epidemiológico sobre a doença aponta queda na taxa de detecção de Aids no país desde 2012. Brasília, 2020. Disponível em: https://www.gov.br/saude/ptbr/assuntos/noticias/casos-de-aids-diminuem-no-brasil. Acessado em 20.Out.2021.

10. MINISTÉRIO DA SAÚDE. Departamento de condições de doenças crônicas e Infecção sexualmente transmissíveis. Manejo do HIV na Atenção Básica. Brasília, 2021b. Disponível em: http://www.aids.gov.br/pt-br/gestores/manejo-dohiv-na-atencao-basica. Acessado em: 30 ago. 2021.

11. MINISTÉRIO DA SAÚDE. Secretaria de Vigilância em Saúde. Departamento de Vigilância, Prevenção e Controle das Infecções Sexualmente Transmissíveis, do HIV/Aids e das Hepatites Virais. Manual Técnico para o Diagnóstico da Infecção pelo HIV em Adultos e Crianças. 4. ed. Brasília - DF, Brasil, 2018. 149 p.: il. Disponível em: file:///C:/Users/USER/Downloads/manual_tecnico_hiv_27_11_2018_web.pdf. Acessado em: 22 Ago. 2021.

12. MINISTÉRIO DA SAÚDE. Secretaria de Atenção à Saúde. Departamento de Ações Programáticas e Estratégicas. Proteger e cuidar da saúde de adolescentes na atenção básica. 2. ed. Brasília: Ministério da Saúde, 2017a. Disponível em:https://bvsms.saude.gov.br/bvs/publicacoes/proteger_cuidar_adolescentes_atencao_basica_2ed.pdf. Acessado em: 30 Ago. 2021.

13. MINISTÉRIO DA SAÚDE. Testes rápidos de HIV e Sífilis na Atenção Básica. Secretaria de Atenção Primária a Saúde. Brasília, 2021a. Disponível em: https://aps.saude.gov.br/ape/cegonha/testerapido. Acessado em: 30 ago. 2021.

14. OLIVEIRA JAS, AFONSO TM. O Teste Rápido para o Diagnóstico de HIV na Atenção Primária à Saúde e a importância da atuação do Enfermeiro. Congresso Internacional de Enfermagem (CIE), 2017; 1(1): e3049.

15. ORGANIZAÇÃO MUNDIAL DE SAÚDE (OMS). Atenção primária à saúde: O que é atenção primária à saúde? Boletim Informativo, 2020. Disponível em: https://www.paho.org/pt/topicos/atencao-primaria-saude. Acessado em 22. ago.2021

16. ORGANIZAÇÃO MUNDIAL DE SAÚDE (OMS). Global strategy on human resources for health: Workforce 2030 [Internet]. 2016. Disponível em: http://apps.who.int/iris/bitstream/handle/10665/250368/9789241511131eng.pdf;jsessionid=039D82637D28A282A11 5BA28402C1E61? sequence $=1$. Acessado em 28. ago.2021

17. PINHO CM, et al. Avaliação das medidas de controle do HIV na atenção básica. Revista Electronica Acervo Saúde, 2020; 12(8):e3462.

18. PORTELA, LMSR, et al. Assistência de enfermagem no pré-natal de soropositivas: uma revisão integrativa. Research, Society and Development, 2021, 10 (2): e28910212265.

19. PREVIATI SM, et al. A importância do aconselhamento no exame rápido de HIV em gestantes durante o pré-natal. Journal of Health Biological Science, 2018; 7(1): 75-81.

20. PROGRAMA CONJUNTO DAS NAÇÕES UNIDAS SOBRE HIV/AIDS (UNAIDS). Estatísticas globais sobre HIV em 2019. 2019. Disponível em: https://unaids.org.br/estatisticas/. Acessado em: 10. set. 2020. 
21. RAMOS FBP, et al. A educação em Saúde como Ferramenta Estratégica no Desenvolvimento de Ações de Prevenção da Transmissão do HIV: Um Relato de Experiência. Revista Eletrônica Acervo Saúde, 2019; Sup.19:e509.

22. SANTOS AHF, et al. Atuação do Enfermeiro na Prevenção de Infecções Sexualmente Transmissíveis na Atenção Básica, Anais dos CONCIFA, 2018; 1 (1): 315-322.

23. SANTOS CP, et al. Vivência das adolescentes e jovens com HIV: um estudo fenomenológico. Revista Oficial do núcleo de estudos da saúde do adolescente /UERJ. 2010; 7 (1): e2010-0320.

24. SANTOS SMP, et al. Roteiros de sexualidade construídos por enfermeiros e a interface com a atenção em infecções sexualmente transmissíveis/HIV. Escola Anna Nery, 2019; 23(4): e20190078.

25. SILVA LMS, et al. Cotidiano de mulheres após contágio pelo HIV/AIDS: subsídios norteadores da assistência de enfermagem. Texto \& Contexto - Enfermagem, 2013; 22(2): 335-342.

26. SOUZA J, et al. Fatores atribuídos a assistência de enfermagem aos portadores da infecção pelo vírus do HIV/AIDS. Revista Eletrônica Acervo Saúde Enfermagem, 2021; 10, e6832.

27. SOUZA LRB, et al. Percepções dos enfermeiros mediante a realização do teste rápido de HIV/AIDS na atenção primária à saúde. Revista Brasileira Multidisciplinar, 2020; 23(2).

28. TUMÉ, E, et al. Formação e prática de enfermeiros para a Atenção Primária à Saúde - avanços, desafios e estratégias para fortalecimento do Sistema Único de Saúde. Saúde debate, 2018; 42(1): 275-288. 